\title{
El verdadero rostro de la informalidad empresarial en el sector turismo del cusco: contraste metodológico y evidencias de su influencia en el desempeño económico.
}

\section{The true face of business informality in the tourism sector in Cusco: methodological contrast and evidence of its influence on economic performance.}

DOI: $10.46932 / \mathrm{sfjdv} 2 \mathrm{n} 2-037$

Received in: january 1st, 2020

Accepted in: March 30th, 2020

\section{Jean Paul Benavente García}

Master en Economía y Políticas Públicas, se desempeña como Docente Principal en la Escuela de Economía de la Universidad San Antonio Abad del Cusco - Perú. Responsable del Proyecto Canon de Investigación: "Impactos de la Actividad Turística en la Región Cusco: Aplicación de la Cuenta Satélite para Impulsar un Turismo Inclusivo y Competitivo".

Correo electrónico: jean.benavente@ unsaac.edu.pe

\section{María Manuela Farfán Qquerari}

Estudiante de la Maestría de Gestión de la Inversión Social de la Universidad del Pacifico - UP, quien se desempeñó como asistente de Investigación en la Universidad San Antonio Abad del Cusco. Articulo desarrollado en el marco del Proyecto Canon de Investigación: "Impactos de la Actividad Turística en la

Región Cusco: Aplicación de la Cuenta Satélite para Impulsar un Turismo Inclusivo y Competitivo".

Correo electrónico: mm.farfanq@alum.up.edu.pe.

\section{RESUMEN}

Este documento tiene como objetivo analizar la influencia de la informalidad sobre el desempeño económico. Para ello, se propone una metodología novedosa para medir la formalidad empresarial usando una base de datos de la Administración Tributaria que permite tener el registro de todas las empresas de la Región Cusco. Según estimaciones por el Método Generalizado de Momentos, así como de modelos de elección discreta. En el que se muestra que la informalidad empresarial en la Región Cusco representa el 56\%; mientras que los ingresos generados de una Micro y Pequeñas Empresas (Mipyme) informal se traduce en una brecha $26 \%$ menor al ingreso generado por la empresa formal. Asimismo, la probabilidad de ser una MiPyme informal es más elevada en determinadas actividades económicas en virtud al perfil y características de las empresas.

Palabras clave: Informalidad, actividades turísticas, distritos turísticos, desempeño económico, Región Cusco.

\begin{abstract}
The objective of this paper is to analyze the influence of informality on economic performance. For this purpose, a novel methodology is proposed to measure business formality using a database of the Tax Administration that allows to have the registry of all the companies in the Cusco Region. According to estimations by the Generalized Method of Moments, as well as discrete choice models. In which it is shown that business informality in the Cusco Region represents 56\%; while the income generated by an informal Micro and Small Business (MSME) translates into a gap 26\% lower than the income generated by the formal company. Likewise, the probability of being an informal MSME is higher in certain economic activities due to the profile and characteristics of the companies.
\end{abstract}

Key words: Informality, tourism activities, tourism districts, economic performance, Cusco Region. 


\section{INTRODUCCIÓN}

La informalidad en el sector turismo tiene una alta incidencia y constituye una de los principales obstáculos al momento de establecer políticas para mejorar la prestación de sus servicios (MINCETUR, 2016). Este problema surge cuando los costos de circunscribirse al marco legal y normativo de un país son superiores a los beneficios, ya que involucra costos tanto en términos de ingresar a este sector (largos, complejos y costosos procesos de inscripción y registro), como en términos de permanecer dentro del mismo (pago de impuestos, cumplir las normas referidas a beneficios laborales y remuneraciones, manejo ambiental, salud, entre otros) Loayza (1999). Del mismo, De Soto (1989) consideran la informalidad; como aquellas fuerzas que mantienen a las personas dependientes de las economías subterráneas son las barreras burocráticas a la propiedad legal y la falta de estructuras legales que reconocen y fomentan la propiedad de los activos. Mientras que, Bromley (1998) de la tesis de De Soto, desprende que no son los individuos informales sino sus actividades y que la mayoría de los casos de las unidades económicas desobedecen disposiciones legales precisas.

En relación con ello, los diversos estudios estimaron la informalidad empresarial y/o sectorial a nivel del Perú, como el Instituto Nacional de Estadística e Informática (INEI) a través de la Encuesta Nacional de Hogares (en adelante, ENAHO) estima que el porcentaje de unidades productivas informales en Perú al 2016 alcanza un 85.8\%, mientras que en Cusco representan un 88.2\% INEI (2017). Sin embargo, Infante y Chacaltana (2014), muestran una tendencia decreciente de la informalidad en las Micro y Pequeña Empresa (en adelante, Mypes), el cual se redujo de 76\% en 2004, al 60\% en 2012, considerando a las Mypes formales a aquellos que: a) tienen un RUC vigente, b) realizan actividades que generan renta de tercera categoría, c) declaran ventas menores a 1.700 unidades impositivas tributarias (en adelante, UIT) al año, d) están clasificadas como contribuyentes con finalidad lucrativa, y e) tienen una actividad económica declarada de finalidad lucrativa. Asimismo, Tello (2011), concluyo que; las empresas informales (en particular microempresas de 1 a 9 trabajadores, incluyendo trabajadores independientes), las cuales representan el $86.4 \%$ del total de empresas del Perú, los cuales se concentran en agricultura, caza y silvicultura (37.4\%), comercio (23.4\%), servicios (13\%), transporte y comunicaciones (10\%), y diversas ramas manufactureras $(9.1 \%)$.

Respecto a los factores que inciden sobre la decisión de la condición de empresas informales en Perú, se han considerado los estudio de Chahuara y Paucar (2011); quienes identificaron los principales determinantes de la informalidad en Perú, a través del modelo binaria (Logit), identificándose que informalidad en los microempresarios se reduce cuando operan en la rama comercio (frente a servicios o industria), si son de sexo masculino, si dispone (como establecimiento) de servicios de luz y telefonía fija, o si tienen acceso (como hogar) a servicios de internet o telefonía fija. Asimismo, Gustavo Yamada 
(2009), identifica las variables que influyen sobre la informalidad a través de las Regresiones Pool (Mínimos Cuadrados Ordinarios) con información del módulo especializado de "Ingreso del trabajador independiente" en empresas familiares no agrícolas de la ENAHO, entre el 2002 y 2006, tales como: sexo, edad (años), jefe de hogar, estado civil, educación, miembros totales de hogar, teléfono, número de trabajadores. Además, estima que los ingresos tienden a aumentar si se cuenta con un local fijo, una mayor antigüedad, el stock de capital, el número de horas trabajadas, los años de educación del dueño del negocio. Finalmente, el coeficiente positivo de la variable de formalidad (aquellos negocios que cuentan con algún tipo de registro legal, como RUC, RUS o REIR) indica que esta decisión afecta positivamente los ingresos del negocio. Dichos resultados reflejan cambios en la muestra e incluso a una variación en la especificación del modelo a utilizar.

Asimismo, en relación a los beneficios de las empresas informales, la literatura es bastante escaza; por lo que, se consideraron el estudio de Chahuara y Paucar (2011) quienes mencionan que las microempresas formales obtienen mayores beneficios netos que sus pares informales, previo un análisis de los principales determinantes de la informalidad en las Mypes (características personales del microempresario y las características de su negocio que son observables). Además, menciona que la conclusión es limitada en algunos aspectos. Por otro lado, Yamada (2009) estima que los ingresos tienden a aumentar si se cuenta con un local fijo, ya que refleja la facilidad del consumidor de poder encontrar al negocio. Una mayor antigüedad del negocio tiene un impacto positivo y significativo sobre los ingresos del negocio o establecimiento. El stock de capital de las empresas juega un rol importante al tener un efecto directo y positivo sobre los ingresos al igual que el número de horas trabajadas en la empresa. También los años de educación del dueño del negocio tienen un impacto positivo y significativo sobre los ingresos. La experiencia del mismo también aporta de manera directa sobre el incremento de los ingresos; sin embargo, presenta rendimientos decrecientes (el coeficiente cuadrático presenta signo negativo y significativo). También, indica que a los negocios que tienen como propietario a una mujer les va relativamente peor, en la medida que el signo del coeficiente asociado a dicha variable es negativo y significativo. Respecto a los sectores económicos el rubro de producción tiene mejores ingresos que el comercio y servicios. Posteriormente, el coeficiente positivo de la variable de formalidad (aquellos negocios que cuentan con algún tipo de registro legal llámase RUC, RUS o REIR) indica que esta decisión afecta positivamente los ingresos del negocio.

Sin embargo, es importante señalar que la actividad del turismo es una de las más importantes en el Departamento de Cusco, el cual representa más del 15\% del PBI regional, con ello el surgimiento de empresas informales; donde de cada 100 empresas constituidas 50 fracasan en tan solo un año. Además, la informalidad empresarial o sectorial según los estudios realizados por el INEI (2017), señalan que el 
porcentaje de informalidad en Cusco supera el $88 \%$ del total de unidades productivas; cifra que podría ser discutible, por lo excesivo que parece en términos del sector empresarial, sobre todo cuando se configuran sectores económicos importantes como el turismo.

En ese sentido, los estudios abordados permiten entender mejor las definiciones y relaciones de la informalidad empresarial en el Departamento de Cusco, generalmente concentrados en las Microempresa de Subsistencia, Microempresa, Pequeña Empresa y Mediana Empresa (en adelante, MiPymes). En este sentido, este documento plasma un nuevo aporte metodológico de estimar este fenómeno.

\section{METODOLOGÍA}

\subsection{DATOS Y VARIABLES}

El estudio de informalidad empresarial en la Región Cusco está limitado por falta de información empresarial. Dadas estas restricciones, la fuente de información a utilizar en este estudio es la información de la base de datos de los directorios empresariales de SUNAT (2016) del departamento de Cusco, en el que se especifican información de las características básicas de los contribuyentes, tales como condición, estado, régimen, tipo de empresa, CIIU, dirección, ubigeo, ventas netas, costos netos, gastos, impuestos, utilidad, activo, patrimonios con cobertura regional. Dicha base fue complementada con la información obtenida de las encuestas aplicadas en el destino turístico de Cusco en el año de 2016 para el proyecto "Impactos de la Actividad Turística en la Región Cusco: Aplicación de la Cuenta de Satélite para impulsar un Turismo Inclusivo y Competitivo 2015-2016”, en el que se recopiló información de la posición de ruc, permisos sectoriales (DIRCETUR, MUNICIPAL, SANIDAD), tiempo de actividad en el mercado, número de trabajadores, ventas, la integración con otras MiPymes, la actividad que realiza, sexo, nivel de instrucción del conductor de las empresas. Las variables descritas, se utilizaron para los propósitos planteados en el estudio. Las encuestas fueron aplicadas en el destino turístico de Cusco.

Distribución del tamaño de las MiPymes

La distribución del tamaño de MiPymes es de acuerdo a la Ley $\mathrm{N}^{\circ} 30056$, el cual le da facilidades a la inversión, impulsa el desarrollo productivo y el crecimiento empresarial. Asimismo, en el artículo 5 del capítulo II "Medidas para el Impulso al Desarrollo Productivo y al Crecimiento Empresarial”, de la Ley $\mathrm{N}^{\circ} 30056$, se señala que el tamaño de las empresas como: la micro, pequeñas y medianas empresas deben ubicarse en alguna de las siguientes categorías empresariales, establecidas en función de sus niveles de ventas anuales, como se muestra en la Tabla 1.

Tabla 1. Distribución del Tamaño de la Empresa - 2015

\section{TAMAÑO DE LAS MYPES}

Microempresa de Subsistencia (**)

Microempresa

UIT (*)

$<13$ UIT

13-150 UIT

\section{VENTAS ANUALES}

$\mathrm{S} / 51,350.00$

$\mathrm{S} / 51,350.00$ - S/ 592,500.00 
(*) Las unidades impositivas a pagar para el año 2015 son equivalentes a 3950.

Fuente: Elaboración de los autores conforme a la LEY N 30056.

\subsection{CÁLCULO DE LA INFORMALIDAD}

Para el estudio planteamos una metodología alternativa de estimar la informalidad dado que las metodologías del INEI (2016), Yamada (2009), Infante y Chacaltana (2014), D. Tello (2011); quienes estimaron a las unidades productivas informales considerando a las no inscritas en la administración tributaria (SUNAT) y utilizando la Encuesta Nacional de Hogares. Los valores estimados por INEI (2017), D. Tello (2011); alcanzan porcentajes por encima del 80\% de informalidad “sectorial” en la Región Cusco, el cual, es un cálculo excesivo, dado que, si existen más de 165,044 empresas formales registradas en SUNAT que representan el 20\%, estaríamos asumiendo que el otro $80 \%$ (o sea aproximadamente 660,000 empresas) son informales; llegando a un total 825,220 mil empresas en la economía cusqueña. Esto está por encima del número de Población Económicamente Activa (761 mil personas). En relación a ello, se estaría asumiendo que cada trabajador representa más de una unidad económica. En relación con ello, en este estudio se plantea una propuesta metodológica que se aproxima al valor estimado propuesto por Infante y Chacaltana (2014). En ese sentido, consideramos que, para estimar el tamaño de la informalidad empresarial, sería importante partir en definir, que la informalidad no solamente supone "no" tener la posición del RUC, dado que; cualquier pregunta que se realice respecto a la posición del RUC, podrá encontrar sesgos de información. Sin embargo, sería importante considerar la información del "estado" y la "condición" del contribuyente dentro del directorio empresarial el cual permitiría acercarse de mejor forma a la información de la informalidad empresarial, el ingreso por ventas (desempeño económico de las empresas) o al de sus activos de las empresas.

En tal sentido, la metodología propuesta, considera a una Mipyme como formal cuando adquiere un estado de contribuyente "activo" y una condición de contribuyente "habido", "pendiente", "no hallado", criterios que fueron establecidos por expertos de la SUNAT de Cusco. No obstante, las unidades económicas MiPymes que no cumplen con el estado y la condición de la contribuyente mencionada serán consideradas como informales ante el registro de la SUNAT.

\subsection{LA ESTRATEGIA ECONOMÉTRICA}

Nivel 1: Para estimar la influencia de la informalidad en el desempeño económico empresarial se plantea el Modelo Lineal Generalizado $(M L G)$. Considerando como variable dependiente al logaritmo de las ventas anuales de dichas empresas y la informalidad empresarial como variable objetivo del análisis: 


$$
\log \left(\operatorname{ventas}_{\text {anuales }}\right)=\beta_{0}+\beta_{1} \operatorname{Informalidad}_{\mathrm{i}}+\mu_{\mathrm{i}}
$$

Por lo tanto, $\beta_{1}$ se define como: $\beta_{1=}\left(E\left(\log \left(\right.\right.\right.$ ventas anuales $\left.\left.\left._{i}\right)\right)-\beta_{2} E\left(\operatorname{Informalidad}_{\mathrm{i}}\right)\right)$. Sin embargo, las ventas anuales también dependen de otros factores. El cual presenta un estimador de MICO de $\beta=\left(\beta_{0}, \beta_{1}\right)^{\prime}$ no sea consistente.

Para poder obtener un estimador consistente de $\beta$ incorporamos unas variables de control que permitirán resolver este problema. Estas son: activos de empresa que expresa su capacidad, distrito o ubicación de la empresa, dado que donde hay más aglomeración de empresas hay probabilidad de mayor información y actividad turística, que por ser una actividad que atrae la participación de empresas, también genera relación con la informalidad.

$\log \left(\right.$ ventas anuales $\left._{i}\right)=\beta_{0}+\beta_{1}$ Informalidad $_{\mathrm{i}}+\beta_{2}$ activos $_{\mathrm{i}}+\beta_{3}$ distrito $_{\mathrm{i}}+$

$$
\beta_{4} \text { Actividades económicas características }_{\mathrm{i}}+\mu_{\mathrm{i}}
$$

Incluso incorporando estas variables, la exogeneidad de la informalidad no está garantizada. Es posible que la relación sea del otro sentido también. Es decir, las ventas anuales pueden afectar el estado de formalidad de las empresas. Esto es, a mayor venta, mayor probabilidad de ser formal. Es así que se propone un instrumento que afecta directamente la informalidad. Este instrumento se vincula con la oficina territorial de SUNAT en los distritos de Cusco, con la finalidad de sensibilizar, fiscalizar, etc., la informalidad, y donde además este instrumento no tiene un efecto sobre la variable dependiente o ventas de las empresas. Al instrumento lo denominamos como agencias ubicadas en el distrito $\mathrm{k}$.

\section{$\mathrm{Z}=$ Agencias $_{\mathrm{k}}$}

Volviendo al ejemplo anterior, la cantidad de agencias de un distrito no va a depender de la motivación de los empresarios. Sin embargo, siempre es cierto que la formalidad de las empresas este muy correlacionada con las cantidades de agencias de la SUNAT. En otras palabras,

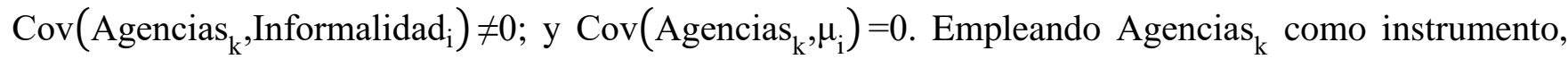
obtenemos estimadores consistentes de $\beta_{0}$ y $\beta_{1}$. De tal modo que:

$\operatorname{Cov}\left(\log\right.$ Venta $_{\text {Anuales }_{i}}$ Informalidad $\left._{i}\right)=$ 


$$
\beta_{1} \operatorname{Cov}\left(\text { Agencias }_{k} \text { Informalidad }_{\mathrm{i}}\right)+\operatorname{Cov}\left(\text { Agencias }_{\mathrm{k}}, \mu_{\mathrm{i}}\right)
$$

Finalmente, en términos econométricos, la forma funcional del modelo de logaritmo de ventas anuales de las empresas está representada de la siguiente forma:

$$
\log \mathrm{Y}_{\mathrm{i}}=\propto_{\mathrm{i}}+\beta_{\mathrm{k}} \widehat{X}_{\mathrm{l}}+\sum_{\mathrm{i}=1}^{\mathrm{n}} \delta_{\mathrm{k}} \mathrm{C}_{\mathrm{i}}+\mu_{\mathrm{i}}
$$

$\widehat{\boldsymbol{X}_{\boldsymbol{\imath}}}$ : Informalidad empresarial estimada; Dummy; Informal “1”, Formal “0”. $\mathbf{C}_{\mathbf{i}}$ : Vector de las variables de control relacionado con las características de las empresas i, que incluye el total de activos de las empresas, actividades económicas características de la Mype, localización de la Mype respecto al territorio (Turístico y no Turístico) y $\boldsymbol{\mu}_{\mathbf{i}}$ : Es el término no sistémico, no observable.

Nivel 2: Regresión Probit, para identificar los principales factores que inciden en la decisión de formar parte del sector informal (MiPyme) del sector turismo de la Región Cusco, se utilizó la regresión probit, dado que es una regresión de respuesta binaria que permite identificar la probabilidad de que un evento ocurra o no. Por consiguiente, la probabilidad de que una MiPyme sea formal va depender de una serie de variables observables y no observables, cuya variable dependiente toma el valor de 1 si el negocio es formal y 0 si el negocio es informal.

En ese sentido, la variable latente, es decir, la probabilidad de que una Mipyme sea informal dependerá de un conjunto de variables explicativas cualitativas y cuantitativas. Tal es así, que el modelo tiene la siguiente expresión:

$$
\mathrm{Y}_{\mathrm{i}}=\varnothing\left(\mathrm{X}_{\mathrm{i}} \beta\right)+\mu_{\mathrm{i}}=\varnothing\left(\mathrm{Z}_{\mathrm{i}}\right)+\mu_{\mathrm{i}}
$$

Conocidos los valores de la variable $\mathrm{X}_{\mathrm{i}}$ (características y perfil del empresario MiPyme) se asigna la probabilidad de que una MiPyme sea informal.

$$
\operatorname{Prob}\left(\mathrm{Y}_{\mathrm{i}}=\frac{1}{\mathrm{X}_{\mathrm{i}}}\right)=\mathrm{P}_{\mathrm{i}}
$$

Para los mismos valores identificados en $\mathrm{X}_{\mathrm{i}}$ (las características y perfil de perfil del empresario MiPyme), la probabilidad de que la variable $Y_{i}$ valga cero (MiPyme sea formal) es (1- $\left.P_{i}\right)$, ya que la suma de ambas probabilidades debe ser igual a la unidad. Para tal efecto se tiene la siguiente expresión: 


$$
\operatorname{Prob}\left(\mathrm{Y}_{\mathrm{i}}=\frac{0}{\mathrm{X}_{\mathrm{i}}}\right)=\left(1-\mathrm{P}_{\mathrm{i}}\right)
$$

El fundamento de la regresión está dado en:

$$
\begin{aligned}
& \mathrm{P}\left(\mathrm{Y}=1 \mid \mathrm{X}_{1}, \mathrm{X}_{2}, \ldots \mathrm{X}_{\mathrm{p}}\right)=\mathrm{P}\left(\beta_{0}+\beta_{1} \mathrm{X}_{1}+\beta_{2} \mathrm{X}_{2}+\ldots \beta_{\mathrm{i}} \mathrm{X}_{\mathrm{i}}+\varepsilon\right) \\
& \text { Donde, } 0<\mathrm{P}\left(\beta_{0}+\beta_{1} \mathrm{X}_{1}+\beta_{2} \mathrm{X}_{2}+\ldots \beta_{\mathrm{i}} \mathrm{X}_{\mathrm{i}}+\varepsilon\right)<1
\end{aligned}
$$

Posteriormente, cuando se analiza la distribución de manera porcentual de las MiPymes informales influenciados por un grupo de variables del perfil y características de las Mypes se tiene la siguiente:

\section{Especificación del Modelo:}

$$
\begin{gathered}
\mathrm{P}\left(\left(\mathrm{Y}_{\mathrm{i}}=1 \mid \mathrm{X}_{1 \mathrm{i}} \mathrm{X}_{2 \mathrm{i}} \mathrm{X}_{3 \mathrm{i}} \mathrm{X}_{4 \mathrm{i}} \mathrm{X}_{5 \mathrm{i}} \mathrm{X}_{6 \mathrm{i}} \mathrm{X}_{7 \mathrm{i}} \mathrm{X}_{8 \mathrm{i}}\right)\right)= \\
\mathrm{P}\left(\mathrm{B}_{0}+\mathrm{B}_{1} \mathrm{X}_{1}+\mathrm{B}_{2} \mathrm{X}_{2}+\mathrm{B}_{3} \mathrm{X}_{3}+\mathrm{B}_{4} \mathrm{X}_{4}+\mathrm{B}_{5} \mathrm{X}_{5}+\mathrm{B}_{6} \mathrm{X}_{6}+\mathrm{B}_{7} \mathrm{X}_{7}+\mathrm{B}_{8} \mathrm{X}_{8}+\mathrm{B}_{9} \mathrm{X}_{9}+\varepsilon_{\mathrm{i}}\right)
\end{gathered}
$$

Dónde:

$\boldsymbol{Y}$ : Informalidad: “1” si Mipyme es informal y “0” si la Mipyme es formal. $\mathbf{X}_{\mathbf{1}}$ : Número de trabajadores. $\mathbf{X}_{2}$ : Integración de a Mipyme: "1" (Integrada) y "0" (No está integrada). $\mathbf{X}_{\mathbf{3}}$ : Sexo del conducto de la Mipyme: "1” (varón) y “0” (mujer). $\mathbf{X}_{4}$ : Nivel de Educación del Empresario Mipyme: "1” empresario con educación superior y "0" empresario con educación básica. $\mathbf{X}_{\mathbf{5}}$ : Tipo de Establecimiento: "1" establecimiento propio y "0" establecimiento no es propio. $\mathbf{X}_{\mathbf{6}}$ : Tiempo en la Actividad. $\mathbf{X}_{\mathbf{7}}$ : Micro Empresa. $\mathbf{X}_{\mathbf{8}}$ : Pequeña y Mediana Empresa (Pyme). $\mathbf{X}_{\mathbf{9}}$ : Actividades vinculadas con el Turismo. $\boldsymbol{\varepsilon}$ : error estadístico.

\section{RESULTADOS}

\subsection{ANÁLISIS DESCRIPTIVO DE LA INFORMALIDAD SECTORIAL DE LA REGIÓN CUSCO}

De acuerdo al Directorio Empresarial de SUNAT (2016), en la Región Cusco se ha identificado el registro de 459,089 unidades económicas, de los cuales 65\% $(294,089)$ unidades económicas tienen el registro de personas naturales "sin negocio" y 36\% $(165,044)$ representan a los registros con personería jurídica "con negocio"1.

\footnotetext{
${ }^{1}$ Están constituidas por el tipo de contribuyente; es decir, persona natural con impuestos de $3^{\text {a }}$ categoría, sociedad conyugal, sucesión indivisa, personas jurídicas y sociedades irregulares o cualquier otra forma colectiva de constitución.
} 
En relación a las unidades económicas registradas con personería jurídica, se evidencia que la Región Cusco, tiene un aproximado de 68.4\% unidades económicas de tamaño de microempresas de subsistencia y el $23.8 \%$ las microempresas, cuyas ventas anuales estarían fluctuando entre 13 UIT a 150 UIT. Sin embargo, el estrato de las Pequeñas y Mediana Empresas (en adelante, PyMe) presenta una participación inferior con $7.1 \%$ las pequeñas empresas, $0.2 \%$ las medianas empresas y $0.5 \%$ la gran empresa.

Asimismo, considerando la metodología propuesta de la informalidad evidenciamos que el número de unidades económicas con personería jurídica "con negocio" formal, es decir, la MiPymes formales en la Región Cusco asciende a 44.2\%, mientras que las empresas informales representan un 55.6\%, este último está constituido por aquellas empresas o MiPymes que no cumplen con la condición de formalidad (activo, habido, pendiente y no hallado).

Por lo que, la informalidad empresarial y/o sectorial presenta una relación inversa en relación al tamaño de las MiPymes, en el que aproximadamente el $67 \%$ de las empresas de subsistencia son informales. No obstante, en la microempresa, pequeña, mediana y gran empresa la informalidad es de un aproximado de $34.7 \%$, 19.6\%, 6.5\% y 16.8\%, respectivamente. Cabe señalar, que dichos resultados muestran la necesidad de plantear normas y reglamentos en beneficio de las empresas de menor tamaño a fin de garantizar su permanencia en el mercado y con ello garantizar la posibilidad de desarrollarse; cuyo detalle se muestra en la Tabla 2.

Tabla 2. Distribución de las empresas por formalidad según el estrato empresarial en Cusco

\begin{tabular}{lccccc}
\hline \multicolumn{1}{c}{ Estrato empresarial } & $\begin{array}{c}\mathbf{N}^{\mathbf{0}} \mathbf{d e} \\
\text { empresas } \\
\text { Informales }\end{array}$ & $\mathbf{\%}$ & $\begin{array}{c}\mathbf{N}^{\mathbf{0}} \text { de } \\
\text { empresas } \\
\text { Formales }\end{array}$ & $\begin{array}{c}\text { Total de } \\
\text { Empresa }\end{array}$ & $\begin{array}{c}\text { \% } \\
\text { Microempresa de Subsistencia }\end{array}$ \\
Microempresa & 75,703 & $67.00 \%$ & 37,234 & $33.00 \%$ & 112,936 \\
Pequeña Empresa & 13,641 & $34.70 \%$ & 25,713 & $65.30 \%$ & 39,354 \\
Mediana Empresa & 2,284 & $19.60 \%$ & 9,382 & $80.40 \%$ & 11,666 \\
\hline Total de MiPymes & 20 & $6.50 \%$ & 288 & $93.50 \%$ & 308 \\
\hline Gran Empresa & $\mathbf{9 1 , 6 4 8}$ & $\mathbf{5 5 . 8 0 \%}$ & $\mathbf{7 2 , 6 1 6}$ & $\mathbf{4 4 . 2 0 \%}$ & $\mathbf{1 6 4 , 2 6 4}$ \\
\hline \multicolumn{1}{c}{ Total } & 131 & $16.80 \%$ & 649 & $83.20 \%$ & 780 \\
\hline
\end{tabular}

Fuente: Elaboración de los autores con datos del Directorio de SUNAT - 2015 y 2016. 


\subsection{INFORMALIDAD EN LA EMPRESAS DEL SECTOR TURISMO}

La informalidad en el sector turismo de la Región Cusco está determinado por la clasificación la Organización Mundial del Turismo $(1999)^{2}$ de los productos turísticos, es así que de las 164,264 Unidades Económicas con el registro de personería jurídica, se ha identificado un aproximado de 43\% (71,061 MiPymes) realizan actividades no vinculadas al turismo y 57\% (93,203 MiPymes) efectúan actividades vinculadas con el turismo ya sean de tipo característica y/o conexa y/o no específica. Cabe señalar, que en las actividades vinculadas con el turismo la informalidad en las MiPymes alcanza a 57\% en relación a $55 \%$ en las MiPymes no vinculados al turismo.

Asimismo, la informalidad en las MiPymes que realizan actividades vinculadas con el turismo en la Región Cusco, se ha identificado una informalidad empresarial de $58 \%$ en las actividades No Especificas, $58 \%$ en las Conexas y $54 \%$ en las características, de este último, podemos señalar que en aquellas MiPymes que realizan actividades en el rubro de Agencias de Viajes, la informalidad asciende a $65 \%$, con ello siendo uno de las actividades con mayor porcentaje de la informalidad en relación a los hoteles, restaurantes y otras actividades características, el detalle se muestra en la

Tabla 3.

De acuerdo a la información precedente, se recomendaría que las acciones de control de la informalidad deberían estar direccionadas a aquellas empresas que realizan actividades relacionadas de forma directa o indirecta con los visitantes nacionales o extranjeros para prestar servicios de calidad. Además, se evidencia que la informalidad en agencias de viajes es superior respecto a los restaurantes y hoteles en la Región Cusco.

Tabla 3 Distribución de la Informalidad de las Mipymes por actividades económicas en Cusco.

\begin{tabular}{lccc}
\hline Tipo de Actividades & $\begin{array}{c}\text { Número de Empresas } \\
\text { (MiPymes) }\end{array}$ & \% Formal & \% Informal \\
\hline Actividades No Turísticas & 71,061 & $45 \%$ & $55 \%$ \\
Actividades Turísticas & 93,203 & $43 \%$ & $57 \%$ \\
\hline \multicolumn{2}{c}{ Actividades Turísticas } & & \\
\hline Actividades Conexas & 36,919 & $42 \%$ & $58 \%$ \\
Actividades no Especificas & 27,545 & $43 \%$ & $57 \%$ \\
Actividades Características & 28,740 & $46 \%$ & $54 \%$ \\
\hline \multicolumn{2}{l}{ Actividades Características } & & \\
\hline Hoteles & 3,406 & $62 \%$ & $38 \%$ \\
Restaurantes & 16,617 & $45 \%$ & $55 \%$ \\
Agencias de Viaje & 3,462 & $35 \%$ & $65 \%$ \\
Otras Act. Características & 5,255 & $43 \%$ & $57 \%$ \\
\hline
\end{tabular}

Fuente: Elaboración de los autores con datos del Directorio de SUNAT - 2015 y 2016.

\footnotetext{
${ }^{2}$ Existen tres tipos de productos turísticos. 1) Productos que sin consumo turístico dejarían de existir en cantidades importantes; ejemplos: transporte aéreo regular de pasajeros, alojamiento en hotel, servicios e agencias de viaje (Características). 2) Productos que sin turismo se ven afectados de forma importante o son importantes para el turismo, independientemente del nivel de uso turístico del producto: ejemplos: transporte en taxi, instalaciones deportivas para el espectador (Conexas). 3) Productos que pueden consumir los visitantes, pero tienen poca importancia para el turismo, y el turismo tiene poca importancia para el producto; ejemplos: libros, cosméticos, periódicos, productos farmacéuticos (No Específicos).
} 
Este hecho refuerza la idea de que la informalidad es mucho más prevalente en los negocios que realizan alguna actividad relacionada con el turismo. Por tanto, las acciones de política deben ir dirigidas a su reducción de informalidad de este segmento. Asimismo, la información muestra la elevada muestra de participación de las empresas informales en las empresas de subsistencias, micro y pequeña empresa.

Informalidad en las MiPymes por la ubicación de los distritos de Cusco

Respecto al análisis de la informalidad de la MiPymes por la ubicación de los distritos de Cusco, se ha evidenciado que de 164,264 MiPymes de Cusco, 86\% $(142,138)$ realizan sus actividades en aquellos distrititos considerados como turísticos mientras que $14 \%$ (22,906) están localizadas en los distritos no turísticos, esta evidencia señala que los distritos turísticos son los principales acogedores para el desarrollo de empresas de diferentes tamaños que a diario se constituyan, con probabilidades de ser informal (infraestructura rudimentaria, mala calidad de servicio y sin calidad de innovación) generando una menor productividad.

La informalidad en las MiPymes localizados en los distritos turísticos son superiores en 56\% respectos a los distritos no turísticos que representan 52\%. No obstante, la informalidad en microempresas de subsistencias es decir negocios con ventas anuales menores a 13UITs presentan porcentajes de informalidad superior a las empresas de tamaño micro, pequeña y mediana empresa en los distritos turísticos y no turísticos. Este hecho es muy preocupante ya que la gran cantidad de empresas y negocios que se concentran en este tamaño, el cual requiere lineamiento de política tributaria fundamental para reducir la informalidad en las MiPymes, como se muestra en la Tabla 4.

Tabla 4 Distribución la Informalidad en la MiPymes por la ubicación de los Distrito del Departamento de Cusco

\begin{tabular}{lccc}
\hline $\begin{array}{c}\text { Ubicación de los Distritos del } \\
\text { Departamento de Cusco }\end{array}$ & $\begin{array}{c}\text { Número de Empresas } \\
\text { (MiPymes) }\end{array}$ & \% Formal & \% Informal \\
\hline Distritos no Turísticos & 22,906 & $48 \%$ & $52 \%$ \\
Distritos Turísticos $^{3}$ & 142,138 & $44 \%$ & $56 \%$ \\
\hline Total de las MiPymes & $\mathbf{1 6 4 , 2 6 4}$ & $\mathbf{4 4 \%}$ & $\mathbf{5 6 \%}$ \\
\hline
\end{tabular}

Fuente: Elaboración de los autores con datos del Directorio de SUNAT - 2015 y 2016.

Asimismo, la informalidad por tamaño de las MiPymes en los Distritos Turísticos, es superior en las Microempresas de Subsistencia alcanzando a 68\%, respecto a las Microempresas y Pequeñas empresas que representa $35 \%$ y $20 \%$, respectivamente de los Distritos no Turísticos. Sin embargo, en aquellas empresas de tamaño Mediano la informalidad en los Distritos no Turísticos es superior, que representa $31 \%$ respecto a aquellos los ubicados en los Distritos Turísticos que representan el $4 \%$, esto puede ser

\footnotetext{
${ }^{3}$ Distritos Turísticos. En el estudio se consideraron a 47 Distritos de la Región Cusqueña; de acuerdo a la ubicación de un Distrito dentro de un corredor, circuito, red, espacio y destino turístico. Es decir, si genera un flujo importante de turistas extranjeros y/o nacionales >500 visitantes. Para el año de 2015.
} 
explicado, por la presencia de las Oficinas de SUNAT quienes vienen realizando labores de sensibilización, monitoreo y otros en territorios de mayor aglomeración de empresas, puesto que serían los principales que contribuyen a la recaudación de impuestos, no obstante, estaría pendiente la identificación de aquellas empresas de tamaño mediano en territorios donde la actividad turística del departamento de no Cusco no es tan significativo en la Economía.

Tabla 5 La Informalidad por tamaños de las MiPymes por la ubicación de los Distrito del Departamento de Cusco

\begin{tabular}{|c|c|c|c|}
\hline \multirow{2}{*}{ Tamaño de las Empresas } & \multirow{2}{*}{$\begin{array}{l}\text { Número de } \\
\text { Empresas } \\
\text { (MiPymes) }\end{array}$} & \multicolumn{2}{|c|}{ \% MiPymes Informales } \\
\hline & & Distritos No Turísticos & Distritos Turísticos \\
\hline Micro empresa de Subsistencia & 112,936 & $63 \%$ & $68 \%$ \\
\hline Microempresa & 39,354 & $31 \%$ & $35 \%$ \\
\hline Pequeñas Empresas & 11,666 & $16 \%$ & $20 \%$ \\
\hline Medianas Empresa & 308 & $31 \%$ & $4 \%$ \\
\hline Total de las MiPymes & 164,264 & $44 \%$ & $56 \%$ \\
\hline
\end{tabular}

Fuente: Elaboración de los autores con datos del Directorio de SUNAT - 2015 y 2016.

\subsection{LA INFORMALIDAD Y DESEMPEÑO ECONÓMICO}

Respecto al comportamiento del desempeño económico ${ }^{4}$ y la informalidad por tamaño de empresas, en el estudio de ha identificado que las microempresas de subsistencia informales quienes representan el 67\%, aproximadamente han generando ingresos de ventas promedio de S/. 4,235. Mientras que las microempresas de subsistencia formales logran en promedio S/.10,300 de ingresos por ventas; o sea, 2.43 veces las ventas de las empresas informales. Del mismo modo, la valoración del total de activos que poseen las empresas de susbsistencia informales es de S/. 35,437, en relación a las empresas de subsistencia formales acumularon activos por S/.113,132, evidenciando nuevamente las brechas entre estos dos sectores. Cabe resaltar, que a medida que el tamaño aumenta a microempresa, pequeña y mediana empresa, las brechas de ventas se reducen equiparando las ventas tanto de empresas informales con las empresas formales. El cual se explica porque cuando la empresa es muy pequeña y no alcanza a lograr sus condiciones de formalización entonces está en situación de exclusión, generando ingresos eminentemente de sobrevivencia crónica probablemente muy por debajo de sus costos medios variables. Por su parte, cuando la empresa informal es pequeña o mediana, es probable que esté en situación de escape; es decir, que pudiendo ser formal no lo hace, lo que genera que esta empresa asuma estrategias proclives a evadir o eludir sus compromisos tributarios.

Otro aspecto a diferenciar en el desempeño económico de empresas que se localizan en distritos turísticos o aquellas que se encuentran en distritos no turísticos. En el primer caso, se observa que el

\footnotetext{
${ }^{4}$ El desempeño económico puede ser evaluado por variables como ingreso por ventas, acumulación de activos, costos de producción, gastos de ventas, utilidades, etc., los cuales se encuentran en los estados financieros reportados por las empresas, así como algún perfil adicional del conductor del negocio. No obstante, por la normativa de protección tributaria esta información es reservada
} 
ingreso promedio de ventas es de $\mathrm{S} / .350,841$ para las empresas formales, mientras que para las empresas informales el ingreso promedio es de S/.69,473, expresando una brecha de ingresos de 5 a 1 . Aunque en el distrito no turístico esta brecha es mayor casi de 7 a 1 . Es preciso resaltar que el promedio de ventas en distritos turísticos es menor que el promedio de ventas en distritos no turísticos, así la empresa sea formal o informal. Esto sugiere considerar algunos criterios (cuestión que escapa al alcance del presente artículo): dado que la actividad turística se desarrolla en distritos turisticos y aglomera $86 \%$ de la población de empresas, el tamaño de ingresos promedio será menor. Mientras que en la zona no turística se desarrollan otras actividades extractivas (minería, hidrocarburos, etc.) que contienen solo el 14\% de la población de empresas, con lo que hacen que el tamaño de ingreso promedio sea mayor. Además, se debe advertir que muchas empresas vinculadas al sector turismo y al sector de industrias extractivas no tienen domicilio fiscal en el departamento, lo cual puede generar algunos sesgos de cálculo de ingreso promedio 5 .

Del mismo modo, cuando se analiza el desempeño económico y la informalidad según el comportamiento de la actividades vinculadas al turismo, se puede apreciar que las ventas de las empresas formales son en promedio 12 veces más grandes que las ventas de las empresas informales. Se evidencia además una mayor capacidad de acumulación de activos con lo que se consolida el desempeño económico. Cabe resaltar, que el ingreso promedio de empresas vinculadas en turismo es menor al ingreso promedio de empresas no vinculadas, el cuál es explicado por la mayor población de empresas vinculadas y, por otro, debido al registro de información según domicilio fiscal. No obstante, queda evidencia que tanto en términos territoriales como sectoriales el turismo genera un gran aporte al desempeño económico del departamento de Cusco.

Tabla 6. Distribución de empresas según promedio de ventas y activos en el Departamento de Cusco

\begin{tabular}{|c|c|c|c|c|c|c|}
\hline \multirow{2}{*}{ Estadísticas } & \multirow{2}{*}{$\begin{array}{c}\mathrm{N}^{\circ} \text { de } \\
\text { Empresas }\end{array}$} & \multirow{2}{*}{$\begin{array}{c}\text { \% Empresa } \\
\text { informal }\end{array}$} & \multicolumn{2}{|c|}{ EMPRESAS FORMALES } & \multicolumn{2}{|c|}{$\begin{array}{l}\text { EMPRESAS } \\
\text { INFORMALES }\end{array}$} \\
\hline & & & $\begin{array}{l}\text { Promedio } \\
\text { Ventas }\end{array}$ & $\begin{array}{c}\text { Promedio } \\
\text { Activos }\end{array}$ & $\begin{array}{l}\text { Promedio } \\
\text { Ventas }\end{array}$ & $\begin{array}{l}\text { Promedio } \\
\text { Activos }\end{array}$ \\
\hline \multicolumn{7}{|c|}{ Distribución de Empresas por tamaño de empresas en el Departamento de Cusco } \\
\hline $\begin{array}{l}\text { Microempresa de } \\
\text { Subsistencia }\end{array}$ & $112,936.00$ & $67 \%$ & $10,300.00$ & $113,132.00$ & $4,235.00$ & $35,437.00$ \\
\hline Microempresa & $39,354.00$ & $35 \%$ & $210,053.00$ & $229,872.00$ & $171,145.00$ & $194,369.00$ \\
\hline Pequeña Empresa & $11,666.00$ & $20 \%$ & $1,726,245.00$ & $1,102,095.00$ & $1,353,244.00$ & $496,534.00$ \\
\hline Mediana Empresa & 308 & $7 \%$ & $7,700,107.00$ & $6,069,515.00$ & $8,179,481.00$ & $2,796,006.00$ \\
\hline \multicolumn{7}{|c|}{ Distribución de empresas por Distritos en el Departamento de Cusco } \\
\hline Distrito Turístico & $141,440.00$ & $56 \%$ & $350,841.00$ & $315,200.00$ & $69,473.00$ & $98,448.00$ \\
\hline Distrito No Turístico & $22,824.00$ & $52 \%$ & $611,464.00$ & $941,741.00$ & $88,102.00$ & $72,311.00$ \\
\hline \multicolumn{7}{|c|}{ Distribución de empresas por tipo de actividades económicas en el Departamento de Cusco } \\
\hline $\begin{array}{l}\text { Act. NO Vinculadas } \\
\text { al Turismo }\end{array}$ & $71,061.00$ & $55 \%$ & $696,364.00$ & $764,625.00$ & $121,963.00$ & $93,915.00$ \\
\hline $\begin{array}{l}\text { Act. Vinculadas al } \\
\text { Turismo }\end{array}$ & $93,203.00$ & $57 \%$ & $399,933.00$ & $1,011,699.00$ & $31,889.00$ & $46,973.00$ \\
\hline
\end{tabular}

\footnotetext{
${ }^{5}$ Este tema es ampliamente abordado en el artículo de descentralización fiscal.
} 


\begin{tabular}{lcccccc} 
& & & & & \multicolumn{2}{c}{$\begin{array}{c}\text { SOUTH FLORIDA } \\
\text { JOURMAL OF DEVELOPMENT }\end{array}$} \\
& & & & & & \\
Características & $28,740.00$ & $54 \%$ & $292,095.00$ & $347,821.00$ & $27,163.00$ & $52,233.00$ \\
Conexas & $36,919.00$ & $58 \%$ & $1,014,077.00$ & $4,259,610.00$ & $71,399.00$ & $67,093.00$ \\
No específicas & $27,545.00$ & $57 \%$ & $276,756.00$ & $472,591.00$ & $27,603.00$ & $37,632.00$ \\
\hline
\end{tabular}

Fuente: Elaboración de los autores con datos del Directorio de SUNAT - 2015 y 2016.

\subsection{ESTIMACIÓN ECONOMÉTRICA}

a) La informalidad, el desempeño económico y la contribución de las MiPymes del sector turismo en la Región Cusco

Considerando el desempeño económico como aquellos que está determinado por los ingresos y/o ventas de las empresas, que depende negativamente de la informalidad, es decir, a mayor informalidad menores ventas, esta relación también podría ir en sentido contrario, por lo que se considera un instrumento, a la instalación de agencias de SUNAT, como mecanismo regulador de sesgos o costos/beneficios no observables en dicha relación. Puesto que, la instalación de oficinas de la autoridad tributaria se sitúa en 05 distritos $^{6}$, que contienen una proporción importante de unidades económicas registradas en SUNAT.

En ese sentido, la muestra para la aplicación del modelo fue de 14,767 empresas o unidades económicas localizadas en los 05 distritos, lo cual demuestra la gran aglomeración de empresas donde la reacción espontánea de la creación de empresas obedece a la dinámica económica, generada sobre todo por el crecimiento de actividades extractivas, la actividad turística, el impulso de la construcción debido a la tenencia de recursos canon, expansión de servicios en principales ciudades, etc. Este crecimiento espontáneo conduce también crecimiento de la informalidad. Aunque la autoridad tributaria cumpla con sus objetivos de sensibilización y fiscalización no necesariamente reduce la informalidad en esos distritos, sino más bien trata de equiparar algunos sesgos de información en cuanto a cumplimiento de reglas, de costos de transacción, entre otros, y que no permitan evaluar la influencia unidireccional de la informalidad sobre el desempeño económico.

Po consiguiente, el modelo propuesto de método generalizado de momentos (GMM) de 2 etapas, considera a la informalidad como variable principal del estudio, instrumentada por agencias, el cual genera un efecto negativo significativo sobre las ventas, con coeficiente consistente y robusto, dado que al incorporar variables o cambiar el tamaño de muestra este coeficiente que no varía sustantivamente. En relación con ello, se evidencia que cuando una empresa es informal su desempeño económico es menor en alrededor de $26 \%$, respecto de una empresa formal.

Asimismo, cuando se observa la regresión base tanto la acumulación de activos (que resume la capacidad de la empresa) y la pertenencia al distrito turístico, generan efectos significativos y positivos sobre el desempeño económico. Esto quiere decir que a mayor acumulación de activos las ventas se

\footnotetext{
${ }^{6}$ El departamento de Cusco cuenta con 108 distritos al momento de desarrollo de la investigación. Los distritos son Cusco, San Sebastián, Sicuani, Santa Ana y Espinar.
} 
incrementan, y que, adicionalmente, en tanto una empresa pertenece a un distrito turístico, sus ventas tienden a aumentar. Por tanto, se muestra la importancia de la acumulación de activos (la inversión de las empresas en capital) y sobre todo la importancia del turismo en el destino Cusco.

Por su parte, cuando se incorporan las actividades netamente características del turismo (hoteles, restaurantes y agencias de viajes), también se observa que aumenta el valor esperado condicionado de las ventas.

Finalmente, cuando se divide la muestra en dos (02) sub muestras, como la de las actividades no vinculadas al turismo (8894 observaciones), la informalidad sigue teniendo efectos negativos sobre las ventas, y las variables de control muestran efectos positivos sobre el desempeño económico. Cabe señalar, que la prevalencia de las actividades características, solo aplican para la sub muestra de empresas vinculadas al turismo (5871 observaciones), con lo que el coeficiente de influencia de actividades características se hace más significativo y por tanto su influencia sobre el desempeño económico resulta más consistente, el cual se muestra en la Tabla 6.

Tabla 7. Regresión de método generalizado de momentos (GMM) - Ventas anuales de las empresas respecto a la informalidad, distrito turístico, activos netos y tipo de actividades en la Región de Cusco

\begin{tabular}{|c|c|c|c|c|}
\hline $\begin{array}{l}\text { Logaritmo de Ventas } \\
\text { Anuales - } 2015\end{array}$ & $\begin{array}{l}\text { Regresión } \\
\text { Base }\end{array}$ & $\begin{array}{l}\text { Regresión con } \\
\text { actividades } \\
\text { características }\end{array}$ & $\begin{array}{c}\text { Regresión para Sub } \\
\text { Muestra actividades } \\
\text { vinculadas al turismo }\end{array}$ & $\begin{array}{c}\text { Regresión para Sub } \\
\text { Muestra actividades } \\
\text { No vinculadas al } \\
\text { turismo }\end{array}$ \\
\hline Informalidad Estimada $^{7}$ & -26.973 & -27.009 & -25.29 & -27.049 \\
\hline $\mathrm{Z}$ & $-18.57 * * *$ & $-18.41 * * *$ & $-11.06^{* * *}$ & $-14.97 * * *$ \\
\hline Distrito Turístico $^{8}$ & 1.392 & 1.359 & 1.389 & 1.491 \\
\hline $\mathrm{Z}$ & $4.95 * * *$ & $4.80 * * *$ & $2.65 * *$ & $4.51 * * *$ \\
\hline Total, Activo Neto & $3.48 \mathrm{E}-09$ & $3.52 \mathrm{E}-09$ & $4.18 \mathrm{E}-09$ & 3.79E-09 \\
\hline $\mathrm{Z}$ & $3.18 * * *$ & $3.2 * * *$ & $3.59 * * *$ & 1.51 \\
\hline Características ${ }^{9}$ & --- & 0.368 & 1.07 & --- \\
\hline $\mathrm{Z}$ & --- & 1.27 & $3.51 * * *$ & --- \\
\hline Constante & 13.585 & 13.584 & 12.336 & 13.851 \\
\hline $\mathrm{Z}$ & $30.21 * * *$ & $30.16 * * *$ & $16.16 * * *$ & $25.4 * * *$ \\
\hline Observaciones & 14,765 & 14,765 & 5,871 & 8,894 \\
\hline Root MSE & 11.109 & 11.063 & 10.335 & 11.191 \\
\hline Wald chi2 & $612.37 * * *$ & $608.23 * * *$ & $268 * * *$ & $348.95 * * *$ \\
\hline Stock \& Yogo (4) & 221.53 & 219.25 & 74.046 & 149.05 \\
\hline
\end{tabular}

Fuente: Elaboración de los autores con datos del Directorio de SUNAT - 2015 y 2016.

NOTA: Los símbolos ****, **, * representan significancias estadísticas de $1 \%, 5 \%$ y $10 \%$.

b) Principales factores que inciden sobre la Informalidad empresarial

\footnotetext{
${ }^{7}$ Informalidad estimada está calculada por metodología propuesta donde

${ }^{8}$ Distrito turístico, es la variable control que se refiere a unidades económicas en 47 distritos de 108 donde se desarrolla actividad turística.

${ }^{9}$ Características, indica exclusivamente unidades económicas en hoteles, restaurantes y agencias de viaje.
} 
Para identificar los principales factores que inciden sobre la decisión de formar la condición de informalidad en las empresas del destino turístico de Cusco; en el estudio se estimó una regresión binaria Probit con datos de encuestas aplicadas a las MiPymes, con una muestra representativa de 665 unidades económicas, en el que se tomaron en cuenta las variables relacionadas a las características y perfil de perfil del empresario MiPyme.

En el modelo propuesto, se ha considerado como variable dependiente binaria la informalidad de las MiPymes y como variables independientes el número de trabajadores, integración de las MiPymes, sexo, nivel de instrucción del conductor de la Mipyme, tipo de establecimiento, tiempo en la actividad, tamaño o estrategia empresarial de la Mipyme (Micro y Pyme), y MiPymes vinculados al sector turismo.

De tal manera que la estimación descrita muestra resultados de una significancia global que presenta un p-value menor que 0,05 ; decir, que existe una interrelación de variables explicativas respecto a la expuesta con $\mathrm{R} 2=0.1654$. Respecto a la significancia individual de las variables explicativas, se precisa que las variables como: integración de las MiPymes, sexo, tiempo de actividad, micro empresas, hospedajes, restaurantes, comercio, artesanía, transporte terrestre y transporte turístico presentan una significancia al 99\%, mientras que el nivel de instrucción presenta una significancia de 95\%. Por otro lado, existen algunas variables que no presentan significancia individual tales como: número de trabajadores, tipo de establecimiento y las PYME como se aprecia en el (tabla anexo 3).

Asimismo, fue necesario permitir una explicación adecuada de los resultados, considerando los efectos marginales de cada uno de las variables. Donde la probabilidad de que una MiPymes del destino turístico de Cusco decida ser informal es de $9.6 \%$ para la muestra específica del estudio.

Tabla 8. Efectos Marginales de las estimaciones del Modelo Probit de la Informalidad en las MiPymes (1=Informal y $0=$ Formal)

\begin{tabular}{|c|c|c|c|c|}
\hline \multirow[b]{2}{*}{$Y=\operatorname{Pr}($ informalidad $)=0.09624971$} & \multicolumn{2}{|c|}{ Estimación } & \multicolumn{2}{|c|}{ Efectos Marginales } \\
\hline & $d y / d x$ & Z & $d y / d x$ & Z \\
\hline Número de trabajadores & -0.001 & -0.140 & 0.000 & -0.140 \\
\hline Integración de las MiPymes & -0.407 & $(-2.81)^{* * *}$ & -0.073 & $(2.72)^{* * *}$ \\
\hline Sexo del conductor de la MiPymes & -0.349 & $(-2.40)^{* *}$ & -0.058 & $(2.45)^{* * *}$ \\
\hline Nivel de Instrucción del Conductor de la Mipyme & -0.349 & $(-2.51)^{* * *}$ & -0.063 & $(2.39)^{* *}$ \\
\hline Tipo de establecimiento & 0.096 & 0.63 & 0.017 & 0.620 \\
\hline Tiempo en la actividad & -0.002 & $(-2.87)^{* * *}$ & 0.000 & $(2.87)^{* * *}$ \\
\hline Micro empresa & 0.832 & $(1.72)^{* *}$ & 0.086 & $(3.27)^{* * *}$ \\
\hline Pequeña y mediana empresa (PYMEs) & 0.699 & 0.97 & 0.175 & 0.740 \\
\hline Hospedajes & -1.863 & $(-5.21)^{* * *}$ & -0.153 & $(8.09)^{* * *}$ \\
\hline Restaurantes & -1.34 & $(-5.20)^{* * *}$ & -0.156 & $(6.10)^{* * *}$ \\
\hline Tejido de fibras textiles & -0.606 & $(-2.03)^{* * *}$ & -0.074 & $(2.95)^{* * *}$ \\
\hline Comercio al por menor & -1.242 & $(-4.88)^{* * *}$ & -0.186 & $(5.08)^{* * *}$ \\
\hline Transporte turístico & -1.237 & ()$-2.66)^{* * *}$ & -0.096 & $(6.29)^{* * *}$ \\
\hline Transporte terrestre & -1.843 & $(-3.38) * * *$ & -0.10 & $(7.48)^{* * *}$ \\
\hline Constante & 0.799 & 0.008 & & \\
\hline Número de observaciones: & \multicolumn{2}{|c|}{665} & & \\
\hline Pseudo R2: & \multicolumn{2}{|c|}{0.1615} & & \\
\hline
\end{tabular}


Fuente: Elaboración de los autores con encuestas.

NOTA: Los símbolos *** representa significancia estadística al $1 \%$.

En la estimación de los efectos marginales se evidencia que la probabilidad de que una Mipyme sea informal disminuirá $-7.3 \%$ promedio cuando este se integre con otras empresas y/o negocios; es decir, una articulación de las MiPymes con otras empresas genera menor propensión a que esta sea informal.

Así cuando el conductor de la Mipyme es varón la probabilidad de que una MiPymes sea informal disminuye en promedio un $-5.9 \%$; este resultado es un indicador de una posible diferencia de género en el mercado laboral en destino turístico de Cusco.

Contar con un nivel de educación superior disminuye la probabilidad de que la MiPymes sea informal en $-6.3 \%$ promedio; esto puede darse porque el contar con una educación superior implica tener conocimiento de los beneficios que se puede conseguir si la empresa es formal. Respecto al variable de tiempo en actividad de la MiPymes es una variable que no tiene efectos en la probabilidad de que la MiPymes sea informal o formal; sin embargo, presenta una significancia individual. Las microempresas (microempresa de subsistencia y micro empresa) muestran coeficiente de 0.086 , el cual implica que cuando el negocio tiene ventas anuales inferiores a 1500 UITs, la probabilidad de decidir formar parte del sector informal aumenta en 8.6 puntos porcentuales.

Por último, en la variable MiPymes vinculados al sector turismo se aplicaron los efectos fijos de tal manera que las empresas o negocios de agencias de viajes fue conferida con hospedajes, restaurantes, tejido de fibras textiles, comercio al por menor, transporte turístico y transporte terrestre. Donde la probabilidad de que estas agencias de viajes sean informales respecto a los hospedajes, restaurantes, tejido de fibras textiles, comercio al por menor, transporte turístico y transporte terrestre, disminuirá en -15.3\%, $-15.6 \%,-7.4 \%,-18.5 \%,-9.6 \%$ y $-10.4 \%$, respectivamente. Es decir, que en las agencias de viajes la probabilidad de ser informal será mucho mayor que las demás actividades mencionadas.

\section{CONCLUSIONES}

A partir de la evidencia, la disponibilidad de información y sujetos al carácter todavía exploratorio del esquema metodológico propuesto, podemos sostener que las MiPymes informales tienen menor desempeño económico frente a aquellas que se encuentran en el sector formal; esto conlleva a considerar que existe un beneficio por operar como empresa formal; más aún cuando las empresas se encuentran vinculadas al sector turismo y cuando sus características propias mejoran. Este resultado es consistente, dado que al incorporar variables o cambiar el tamaño de muestra el coeficiente asociado a la informalidad no varía sustantivamente. Los resultados basados en Método Generalizado de Momentos (GMM) con informalidad instrumentada por agencias tributarias, permiten concluir que las MiPymes informales 
representan el 56\% de las empresas localizadas en Cusco y conllevan menores ventas anuales (ingresos) en un promedio de -26\%; esta evidencia se ajusta a los hallazgos obtenidos por Chahuara y Baldeón (2011) y Yamada (2009).Del mismo modo, cuando se analiza el desempeño económico y la informalidad según el comportamiento de la actividades vinculadas al turismo se puede apreciar que las ventas de las empresas formales son en promedio 12 veces más grandes que las ventas de las empresas informales.

Asimismo, se ha identificado que los principales factores que inciden sobre la decisión de operar en el sector formal en el sector turismo. Tal es así que; la probabilidad de operar en la formalidad por parte de una agencia de viajes aumenta cuando esta se integra con otras empresas, o cuando el gerente de la empresa es dirigido por un varón y cuando posee educación superior, sin embargo; la probabilidad disminuye para aquellas empresas cuyo tamaño es menor y tiene mayor tiempo de antigüedad.

Finalmente, se propone que la estimación de la informalidad empresarial no solo sea explicada por la posición de RUC, sino por el análisis de condición y estado del contribuyente que son factores cruciales para entender este problema. El hecho de que una unidad económica con negocio, no se encuentre activa, o no tenga la condición habida, o esté suspendida, genera niveles de informalidad, que influyen en su desempeño económico, dado que sobre todo cuando son MiPymes, siempre estarán realizando alguna actividad económica. Esto contempla impulsar una discusión acerca del esquema utilizado para calcular el sector informal desde el INEI, que en los últimos años viene publicando la Cuenta Satélite de la Informalidad; pues en el presente artículo, sostenemos que los cálculos de informalidad empresarial basados en reportes de las Encuestas de Hogares, están sobreestimados y por tanto, no permite generar metas adecuadas de políticas públicas para enfrentar el problema de fondo de la informalidad. 


\section{BIBLIOGRAFÍA}

Chahuara Vargas, P. R., \& Baldeón Paucar, H. J. (Setiembre de 2011). La informalidad en el microempresario peruano:Determinantes, costos e implicancias de política 2007-2009. Consorcio de Investigación Económica y Social.

- D. Tello, M. (Febrero de 2011). Indicadores del sector Mype informal en el Perú: valor agregado, potencial exportador, capacidad formalizarse y requerimientos de normas técnicas peruanas de sus productos. Departamento de Economía, Pontificia Universidad Católica del Perú.

- $\quad$ De Soto, H. (1986). El Otro Sendero: la Revolucion Informal. Colombia: Printer.

- $\quad$ Infante , R., y Chacaltana, J. (Abril, 2014). Hacia un Desarrollo Inclusivo en el caso del Perú. Santiago de Chile: Comisión Económica para América Latina y el Caribe (CEPAL).

- $\quad$ INEI. (2016) Producción y Empleo Informal en el Perú Cuenta Satélite de la Economía Informe 2007-2015. Lima: INEI. \& INEI. (2017). Producción y Empleo Informal en el Perú Cuenta Satélite de la Economía Informe 2007-2016. Lima: INEI.

- $\quad$ LEY N ${ }^{\circ}$ 30056. (s.f.). Ley que modifica diversas leyes para facilitar la inversión, impulsar el desarrollo productivo y el crecimiento empresarial . Lima.

- $\quad$ Loayza, N. (1999). Causas y consecuencias de la informalidad en el Perú. Banco de Reserva del Perú.

- $\quad$ MINCETUR. (2016). Plan de apoyo a la formalización del sector turismo. Lima: Ministerio de Comercio Exterior y Turismo.

OMT. (1999). Orientaciones generales para la elaboración Cuenta Satélite de Turismo (CST): Medición de la OFERTA TURÍSTICA. Madrid- España.

- $\quad$ Tello, M. (2005-2009). Los efectos goteo (trickle down effects) del crecimiento: un análisis del desempeño económico del sector informal a nivel de regiones en el Perú. Desigualdad distributiva en el Perú: dimensiones.

- $\quad$ Yamada, G. (09 de Enero de 2009). Determinantes del desempeño del trabajador independiente y la microempresa familiar en el Perú. Centro de Investigación de la Universidad del Pacífico. 